\title{
Work Disability in Scleroderma is Greater than in Rheumatoid Arthritis and is Predicted by High HAQ Scores
}

\author{
Janine M. Ouimet, Janet E. Pope*, Iris Gutmanis and John Koval
}

Department of Epidemiology \& Biostatistics, The University of Western Ontario, London, ON, Canada

\begin{abstract}
Objectives: To estimate the frequency of work disability (WD) in a cohort of patients with Systemic Sclerosis (SSc) vs an internal control group of patients with rheumatoid arthritis (RA) with a known high frequency of WD; and to investigate the association between WD and other factors including Health Assessment Questionnaire Disability Index (HAQ-DI) scores, HAQ pain, age, sex, disease duration and education level.

Methods: Cross-sectional data on WD status were obtained from a questionnaire sent to all SSc ( $\mathrm{n}=35$ limited [1cSSc], 26 diffuse [dcSSc]) and a subset of RA patients $(\mathrm{n}=104)$ from a rheumatology practice. WD data, HAQ-DI scores, and demographic/clinical features (age, sex, high school education, disease duration and SSc disease subtype [dcSSc $v s$ $\mathrm{lcSSc}]$ ) were recorded.

Results: The proportion with WD was 0.56 in SSc (95\% CI: 0.43-0.68) vs 0.35 in RA (95\% CI: 0.25-0.44), p=0.009. HAQ-DI scores were significantly higher in work-disabled SSc and RA patients $v s$ those who were employed ( $\mathrm{p}=0.0001$, and $\mathrm{p}<0.0001)$. Multivariate logistic regression analysis demonstrated that higher HAQ-DI scores $(\beta=1.78, \mathrm{p}<0.001)$, disease type (dcSSc, lcSSc, RA) $(\beta=1.32$ for dcSSc, $p=0.032)$, and self-reported disease duration $(\beta=0.04, p=0.042)$ were significantly associated with WD $\left(\mathrm{R}^{2}=0.311\right)$. Adding a work-related factor (self-reported physically demanding work) improved the regression model $\left(\mathrm{R}^{2}=0.346\right)$ and strengthened the HAQ-DI $(\beta=1.86, \mathrm{p}<0.001)$ and $1 \mathrm{cSSc}(\beta=1.24$, $\mathrm{p}=0.024)$ coefficients.
\end{abstract}

Conclusion: The frequency of WD in SSc was high and was greater than in RA. SSc (and dcSSc) had significantly more WD than RA. The HAQ-DI was strongly associated with WD in SSc.

Keywords: Scleroderma, systemic sclerosis, work disability, health assessment questionnaire.

\section{INTRODUCTION}

Work disability (WD) is a common adverse outcome of chronic autoimmune diseases, such as rheumatoid arthritis (RA), which presents an economic burden to society as well as having a negative impact on the well-being of the individual. Systemic sclerosis (SSc) is a relatively rare chronic autoimmune disease which causes fibrosis (hardening) of the skin and potentially many organ systems can become involved causing problems such as lung disease, pulmonary hypertension, digital ulcers and cardiomyopathy to name a few. It seems obvious that SSc patients would have work and functional impairment due to hand deformities resulting from fibrosis and other internal organ involvement. However, despite these complications and the fact that SSc affects people of working age (with a mean age of onset in the $40 \mathrm{~s}$ ), there are currently no research studies of WD secondary to SSc in the literature. Due to the nearly universal involvement of disease manifestations in the hands of patients with scleroderma and the many serious potential complications of the disease, it is likely that WD in SSc is high. With the apparent trend toward improving survival in SSc, WD is becoming an increasingly important outcome in patients living with SSc, and improved knowledge about WD in this disease may be valuable for future interventions.

*Address correspondence to this author at St. Joseph's Health Care, 268 Grosvenor Street, London, Ontario, N6A 4V2, Canada;

E-mail: janet.pope@sjhc.london.on.ca
SSc is a heterogeneous disease with a variable clinical course. In limited cutaneous (lc) SSc, skin changes are restricted to distal to the elbows and/or knees, but may involve the face and neck regions, compared to diffuse cutaneous (dc) SSc in which skin thickening also occurs proximally. The disease course is more progressive and worse overall in dcSSc $[1,2]$. Hand function may be an important determinant of functional disability in SSc. The hand deformity most observed in SSc is the disabling 'claw' type deformity, which manifests as a loss of flexion at the metacarpophalangeal joint (MCP) and contracture at the proximal interphalangeal joint (PIP). Hand deformity in SSc is distinct from that of RA, where RA may result in subluxations and ulnar drift at MCPs, and malalignment at the wrists. In 115 SSc patients, at least moderate functional disability was present in $37.5 \%$ with $1 \mathrm{cSSc}$ and $64 \%$ with dcSSc [3].

Although WD in SSc is not described in the literature, the prevalence and factors associated with WD in RA are well researched. RA is a logical parallel comparison group for this study of WD in SSc because: 1) it is a chronic autoimmune disease that affects people of similar age and sex as SSc, 2) the frequency of WD is known to be high in RA [429], and 3) a substantial body of research on factors predictive of WD in RA exists. Prevalence (or frequency) estimates of WD in RA have ranged from 9-67\% (average disease duration ranged from 0.5-20 years) in cross-sectional studies and $22-90 \%$ (average disease duration ranged from 1-30 years) in longitudinal studies [4-29]. Reasons for WD in RA 
can vary with disease duration. Some with longstanding RA have joint damage but in early RA, there may be a decreased ability to work due to pain, fatigue, acute joint swelling and lack of adaptation to the chronicity of RA [30]. Disability in RA is often measured using the Health Assessment Questionnaire Disability Index (HAQ-DI) [31, 32] as an outcome measure. Disability appears to increase slowly at a relatively constant rate over the course of the disease after an initial dramatic improvement just following diagnosis (likely due to the onset of treatment) [33]. However, the course of disability for an individual cannot be predicted by disease duration [33]. There may be reversible and irreversible disability in RA - the former from disease activity and the latter from damage. In RA, gender appears to have little influence over functional outcome [34].

The Health Assessment Questionnaire Disability Index (HAQ-DI) measures functional disability and is widely used in rheumatologic diseases. HAQ-DI scores range from 0-3, where zero is no functional disability (or pain) and 3 is extreme disability (or pain). There is consensus in the RA literature that high HAQ-DI scores are associated with WD, as all studies that have investigated the HAQ-DI have confirmed the association with WD [6, 8-13, 15-29]. A large study $(n=823)$ reported a baseline mean HAQ-DI score of 1.0 in those with WD; high HAQ-DI scores and baseline pain were the most significant predictors of subsequent WD [19]. Other studies have confirmed that high baseline HAQDI scores (at least $\geq 1.0$ ) were among the most important predictors of WD within the 2-year to 20-year follow-up periods [17, 23, 26, 28, 29]. Cross-sectional studies have also shown higher HAQ-DI scores in work disabled (compared to working) patients [13, 24]. Table 1 summarizes the association between selected factors and WD in SSc vs RA.

The HAQ-DI has been validated for use in SSc and has demonstrated sensitivity over time [35, 36]. Functional disability (assessed by HAQ-DI) may be similar in SSc and RA; however, studies that combine dcSSc and $1 c S S c$ may mask an effect modification by disease subtype. For example, one study reported that $\mathrm{SSc}(\mathrm{n}=24 \mathrm{dcSSc}, 52 \mathrm{lcSSc})$ had less severe HAQ-DI scores compared to an age- and sex-matched RA cohort, but patients with dcSSc had the highest HAQ-DI scores of all three groups (dcSSc, lcSSc and RA) [37]. Thus, patients with dcSSc may have worse functional disability compared to lcSSc and RA.

Functional disability (often defined as HAQ-DI scores $\geq$ 1.0) has been a significantly important determinant of WD in every one of the RA studies reviewed that assessed functional disability [6, 8-13, 15-26]. High baseline HAQ scores (often defined as $\geq 1.0$ ) have been shown to predict the development of WD [8, 22, 23]. Another study found that the mean HAQ scores over the course of the follow-up period were predictive of WD [19]. Thus, there is strong evidence for the predictive value of the HAQ in determining RArelated WD.

The primary objective of this study was to characterize WD in SSc by determining the frequency of WD in a cohort followed by a rheumatologist (clinical expert in SSc), and also to investigate the association between WD and other factors including HAQ-DI, HAQ pain, age, sex, disease duration, and education level. The secondary objectives were to determine if: 1) the frequency of WD and/or the risk factors for WD in SSc were similar to that in RA; 2) the frequency of WD and/or the risk factors for WD were different between the limited and diffuse subtypes of SSc; and 3) an exploratory work-related variable (self-reported physically demanding job) was associated with WD.

\section{MATERIALS AND METHODOLOGY}

This was a cross-sectional study to investigate factors associated with WD in a SSc cohort using secondary data obtained through a survey administered to SSc and RA patients from one rheumatologist's practice. Ethics approval was obtained from the University of Western Ontario. The sampling frame included all consecutive SSc patients who attended the rheumatology clinic at least once during the period from November 2002 to February 2003. Subjects were classified as those with SSc who reported they had WD at the time of the research, and those who had no work disability (nWD). RA patients (frequency matched with the SSc cohort for age and sex) from the same rheumatologist's practice were chosen as a comparison group with a known high prevalence of WD, and known risk factors for WD (high

Table 1. Demographics, WD and HAQ-DI Scores for the SSc vs RA Subjects

\begin{tabular}{|c|c|c|c|c|c|}
\hline $\mathbf{N}$ & $\begin{array}{c}61 \\
35 \mathrm{lcSSc}, 26 \mathrm{dcSSc}\end{array}$ & 104 & - & - & - \\
\hline Work disabled (n) & $55.7 \%(34)$ & $34.6 \%(36)$ & 2.38 & $1.24,4.54$ & 0.009 \\
\hline Age & $52.00 \pm 1.18$ & $51.90 \pm 0.90$ & - & - & 0.95 \\
\hline Less than high school education & $16.4 \%$ & $9.7 \%$ & 1.82 & $0.71,4.67$ & 0.22 \\
\hline HAQ-DI score & $1.01 \pm 0.09$ & $0.86 \pm 0.07$ & - & - & 0.18 \\
\hline HAQ Pain score ${ }^{a}$ & $1.35 \pm 0.10$ & $1.16 \pm 0.08$ & - & - & 0.14 \\
\hline Self-reported physically demanding work ${ }^{b}$ & $45.4 \%$ & $59.6 \%$ & 0.56 & $0.29,1.11$ & 0.12 \\
\hline
\end{tabular}

P-values provided for dichotomous data were obtained using Fisher's exact chi-squared tests. Proportions are provided for dichotomous data, and the mean \pm SEM is provided for continuous measurements. Odds ratio (OR) are provided for SSc relative to RA. Continuous data were tested using independent t-tests assuming unequal variances.

${ }^{\mathrm{a}}$ Data were missing for $7 \mathrm{RA}, 2 \mathrm{dcSSc}$, and 2 lcSSc subjects; ${ }^{\mathrm{b}}$ Data were missing for $10 \mathrm{RA}, 2 \mathrm{dcSSc}$, and 4 lcSSc subjects. 
HAQ-DI scores, age, and to a lesser extent education and disease duration). The proportions with WD in SSc and RA were compared. Bivariate and multiple logistic regression analyses were used to determine if demographic characteristics (age, sex, self-reported disease duration, education) and patient-centred measurements of functional disability (HAQDI) were associated with the outcome (self-reported WD). Thus, RA was chosen as a known internal reference group in this cross-sectional study of the frequency of WD in a SSc cohort, and factors associated with WD in SSc.

A survey assessed demographic and other personal factors, including work status, education level, and disease duration. The questionnaire was validated in a study of accuracy of self-report of medical history in rheumatologic patients [38]. The questionnaire was mailed to all patients with SSc $(n=129)$ who had been seen at the same rheumatology practice over 2 years, and a sample of RA controls $(n=230)$, frequency-matched with SSc for age and sex. Subjects were blinded to the hypothesis. SSc patients met the ACR diagnostic criteria for SSc, or if they did not, they met diagnostic criteria for CREST. That is to say that patients who had limited SSc and did not have digital pits or resorption and no pulmonary fibrosis were included if they met criteria for CREST $[39,40]$. We then divided them into limited or diffuse disease (those being diagnosed with CREST were included in the limited group as in the Leroy criteria [40]). RA patients met the ACR criteria for RA [41].
After two mailings the response rates were $61 \%$ for SSc $(\mathrm{n}=28 \mathrm{dcSSc}, \mathrm{n}=51 \mathrm{lcSSc})$, and $67 \%$ for RA $(\mathrm{n}=155)$. Respondents to the questionnaire were of similar age and sex distributions as non-respondents. In the SSc group only, respondents had a longer mean disease duration compared to non-respondents (12 y vs 7.2 years, $\mathrm{p}=0.01)$. Subjects were asked "Do you work?" with multiple choice options: 1) Yes, 2) Yes, in the home, 3) Retired - not now but used to, 4) Had to stop work due to illness, 5) Never worked. All respondents who answered the question were considered for eligibility in the WD study. Subjects who chose multiple choice option \# 4 (Had to stop due to illness) were considered work disabled. Subjects who had chosen either of option \# 1 (Yes, ie. working) or \# 2 (Yes, working in the home) were considered to not be work disabled and were included in the study. All subjects who reported that they were retired (option \# 3) or that they had never worked (option \#5) were excluded from the WD study. Fig. (1) describes participant selection and inclusion/exclusion criteria. N=61 SSc and n=104 RA patients were included in the WD SSc final database.

The same questionnaire used to collect WD status also included a question that asked if a subject's job was physically demanding. The decision was made to include this exploratory variable in analyses as it was relevant to the research question but had been subject to item non-response (10 observations were missing in RA, 2 in dcSSc, and 4 in lcSSc).

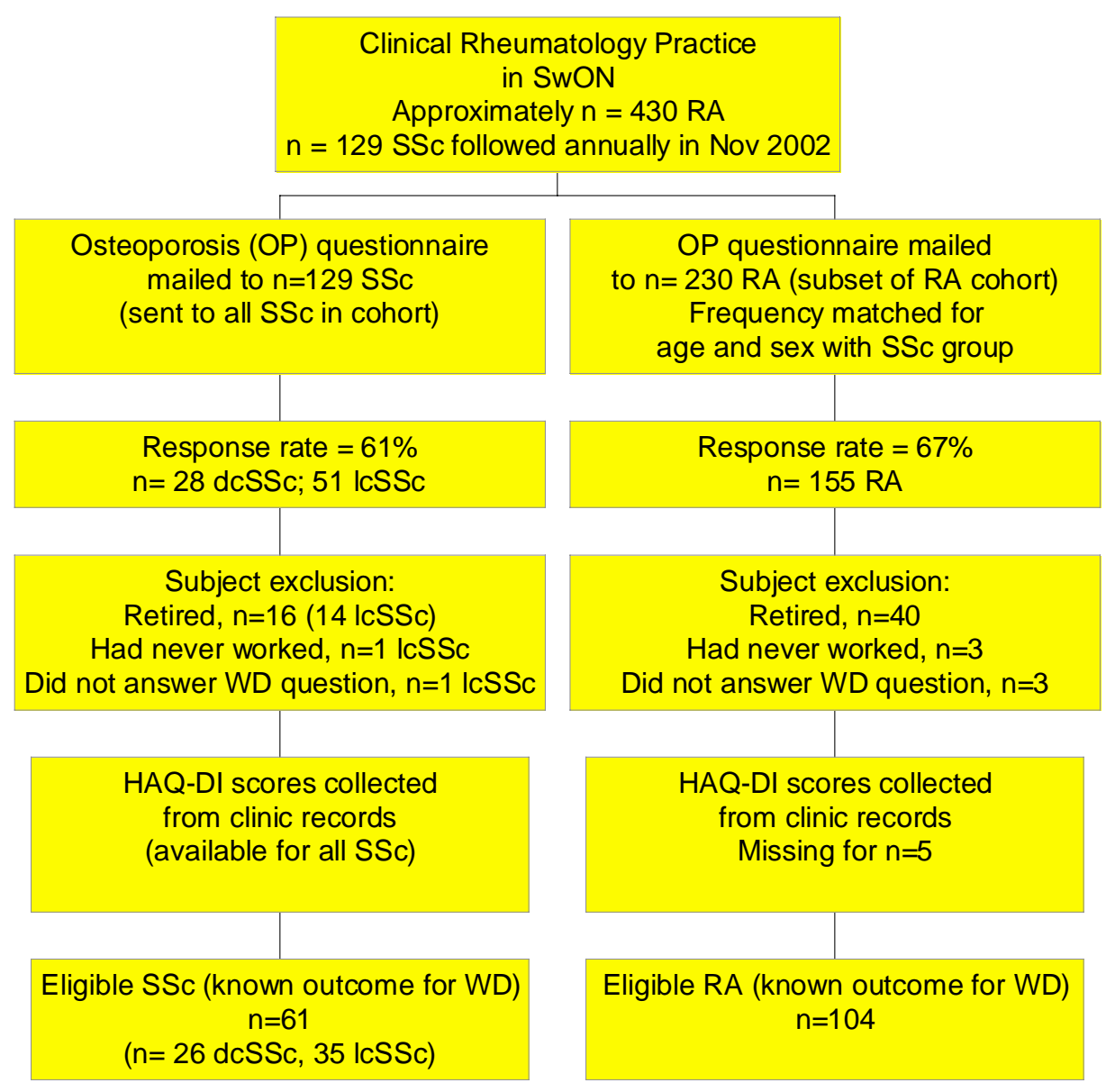

Fig. (1). Eligible patients for WD in SSc and RA analysis. (SwON - Southwestern Ontario). 
As part of the ongoing clinical research initiative at St. Joseph's Health Care London, the HAQ-DI and HAQ pain scales are collected at each clinic visit for all patients. The HAQ-DI that was completed at the clinic visit dated closest to the questionnaire timing was obtained from each subject's medical chart. Consistent with both the SSc and RA literature, a dichotomized HAQ-DI score $\geq 1.0 v s<1.0$ was used. HAQ-DI scores were calculated using the Standard Disability Index Scoring method which takes into account the use of aids and devices (available online at http://aramis.stanford. edu/downloads/HAQ\%20Instructions.pdf). Scores $<1.0$ indicated less functional disability, and scores $\geq 1.0$ indicated functional disability (captures any individual whose activities of daily living (ADLs) can only be carried out 'with some difficulty'). However, the results of the bivariate analyses demonstrated that dichotomizing scores as $<1.0 \mathrm{vs} \geq 1.0$ resulted in a loss of information, and the decision to use continuous variables in the analyses was made. All data were entered into a JMP Version 4 database (SAS Institute, Cary, NC, USA, 2000).

The following statistical analyses were conducted using frequency (and 95\% CI), Student's t-test for continuous variables and Fisher's exact chi-squared test for dichotomous categorical variables. Comparisons of all variables were conducted for all subjects who had reported WD vs nWD at the time of the survey. Comparisons were also conducted for those classified as WD $v s$ nWD within the SSc and RA groups. SPSS version 12.0 (SPSS Inc., Chicago, IL, USA, 2003) was used to construct multivariate logistic regression models of WD on HAQ-DI score along with any other significant explanatory variables (disease type, age, selfreported disease duration, sex, and completion of at least a high school education, and self-report of physically demanding work) using the maximum likelihood method. Subset analyses were conducted between the limited (including those diagnosed with CREST) and diffuse disease subtypes of SSc. Logistic regression models were not constructed for subsets, as the effect of disease subtype was accounted for by the use of the disease type indicator variable.

\section{RESULTS}

\section{Frequency of WD in SSc and RA}

The SSc and RA groups were similar with respect to demographic variables (age, sex, having at least a high school education, and self-reported disease duration) (Table 1). In the study population of 61 subjects with SSc, 34 reported WD, resulting in a frequency proportion of $\mathrm{p}=$ $55.7 \%$; $95 \%$ confidence interval $(43.3 \%, 68.2 \%)$. Thirty-six of the 104 subjects with RA reported WD, resulting in a frequency proportion of $34.6 \% ; 95 \%$ confidence interval $(25.5 \%, 43.8 \%)$. Thus, the frequency of WD in SSc in a southwestern Ontario cohort was significantly higher compared to that in RA ( $56 \%$ vs $35 \%, \mathrm{p}=0.009)$.

\section{WD and Functional Disability (SSc vs RA)}

SSc were significantly more likely than the RA subjects to report WD $(\mathrm{p}=0.009)$. However, SSc patients had only slightly higher mean HAQ-DI and HAQ pain scores (not statistically significant). Despite the higher proportion of WD in the SSc group, the frequency of having a physically demanding job was less common in the SSc compared to RA group (Table 1).

\section{Factors Associated with WD in SSc}

In an analysis of subjects with SSc only, the mean HAQDI score was higher in the work disabled $v s$ not workdisabled subjects $(1.30$ vs $0.66, \mathrm{p}=0.0001)$. Although higher in those with WD, HAQ pain scores and report of physically demanding work were each not significantly different between those with WD and nWD (Table 2).

Disease duration was slightly longer in the WD subjects $(\mathrm{p}=\mathrm{NS})$; however, completion of high school was significantly less common in the WD compared to the nWD group (73.5\% vs $96.3 \%, \mathrm{p}=0.033$ ). Age and female sex showed no significant association with WD status $(\mathrm{p}=0.63$ and $\mathrm{p}=0.49$, respectively). The proportion of subjects with dcSSc (compared to lcSSc) was not significantly different between WD vs nWD (50.0\% in WD vs 33.3\%, $\mathrm{p}=0.21)$. Results are shown in Table 2.

\section{Factors Associated with WD in RA}

In an analysis of subjects with RA only, HAQ-DI scores were significantly higher in the work disabled $v s$ not work disabled subjects (mean 1.42 vs $0.57, \mathrm{p}<0.0001$ ). HAQ pain scores were higher in the work disabled compared to the not work disabled subjects $(1.54$ vs $0.94, \mathrm{p}=0.0002)$, while report of physically demanding work was higher but not significantly different in those with WD compared to nWD $(\mathrm{p}=0.08)$ (Table 2). The proportion of subjects who reported having completed high school was lower in the WD group (85.7\% in WD vs $92.6 \%, \mathrm{p}=0.30$ ); and disease duration was significantly higher in the WD subjects (16.11 years vs 10.58, $\mathrm{p}=0.011)$. Age and female sex showed no significant association with WD status $(\mathrm{p}=0.068$ and $\mathrm{p}=0.41$, respectively). Those with WD had higher (but not statistically significant) HAQ-DI and HAQ pain.

\section{Factors Associated with WD in dcSSc and lcSSc Subsets}

Despite the small number of subjects in the limited and diffuse SSc subsets, analyses were conducted comparing WD and demographic factors between the two groups (Table 3). Self-reported WD was not different between $1 \mathrm{cSSc}$ and dcSSc.

In the dcSSc subset none of the factors were significantly different between those with and without WD. However, those with WD had higher (but not statistically significant) HAQ-DI and HAQ pain scores. The magnitude of the difference in the HAQ-DI scores suggests that there may be a true difference that this study was underpowered to detect. It is also possible that those with WD were different in terms of their demographic characteristics (for example, 29.4\% with WD had not completed high school compared to $0 \%$ with $\mathrm{nWD}, \mathrm{p}=0.13$ ), and may have had a higher frequency of physically demanding work $(\mathrm{p}=0.39$; only $\mathrm{n}=24 \mathrm{dcSSc}$ subjects provided data). However, no conclusions can be drawn based on these underpowered data (Table 4).

The lcSSc subset, however, showed several variables that were associated with WD. The HAQ-DI scores were significantly higher among subjects who were work disabled compared to those who were not work disabled $(p=0.0016$, $\mathrm{p}=0.0015$, and $\mathrm{p}=0.002$, respectively). Demographic charac- 
Table 2. HAQ Scores and Demographic Factors for those who are Work Disabled $v$ s Employed for Each Group of SSc or RA Subjects

\begin{tabular}{|c|c|c|c|c|c|}
\hline & WD & nWD & OR & $95 \% \mathrm{CI}$ for OR & p-Value \\
\hline \multicolumn{6}{|l|}{ SSc Only } \\
\hline $\mathbf{N}$ & 34 & 27 & - & - & - \\
\hline Age & $52.6 \pm 1.72$ & $51.3 \pm 1.93$ & - & - & 0.63 \\
\hline Diffuse cutaneous SSc (dcSSc) & $50.0 \%$ & $33.3 \%$ & 2.00 & $0.70,5.69$ & 0.21 \\
\hline Self-reported disease duration & $11.98 \pm 1.29$ & $9.85 \pm 1.43$ & & & 0.27 \\
\hline Less than high school education & $26.5 \%$ & $3.7 \%$ & 9.36 & $1.10,79.37$ & 0.03 \\
\hline HAQ-DI & $1.30 \pm 0.10$ & $0.66 \pm 0.12$ & - & - & 0.0001 \\
\hline HAQ Pain ${ }^{a}$ & $1.49 \pm 0.14$ & $1.20 \pm 0.16$ & - & - & 0.18 \\
\hline Age & $54.0 \pm 1.45$ & $50.8 \pm 1.06$ & - & - & 0.07 \\
\hline Female sex & $80.6 \%$ & $86.8 \%$ & 0.63 & $0.21,1.87$ & 0.41 \\
\hline Self-reported disease duration & $16.11 \pm 1.72$ & $10.03 \pm 1.22$ & & & 0.008 \\
\hline Less than high school education & $14.3 \%$ & $7.4 \%$ & 2.10 & $0.56,7.81$ & 0.30 \\
\hline HAQ-DI & $1.42 \pm 0.10$ & $0.57 \pm 0.07$ & - & - & $<0.0001$ \\
\hline HAQ Pain ${ }^{a}$ & $1.54 \pm 0.12$ & $0.94 \pm 0.09$ & - & - & 0.0002 \\
\hline Self-reported physically demanding work ${ }^{b}$ & $71.4 \%$ & $52.5 \%$ & 2.26 & $0.92,5.52$ & 0.08 \\
\hline
\end{tabular}

P-values provided for dichotomous data were obtained using Fisher's exact chi-squared tests. Odds ratios (OR) have been provided for work disabled relative to employed. Continuous data were tested using independent t-tests assuming unequal variances.

${ }^{\text {a }}$ Data were missing for $7 \mathrm{RA}, 2 \mathrm{dcSSc}$, and $2 \mathrm{lcSSc}$ subjects; ${ }^{\mathbf{b}}$ Data were missing for $10 \mathrm{RA}, 2 \mathrm{dcSSc}$, and 4 lcSSc subjects.

teristics were similar between groups, with the exception of self-reported disease duration, which was longer in the WD group $(\mathrm{p}=0.04)$. Although slightly higher among those with WD, reported physically demanding work was not significantly different between those who were work disabled compared to not work disabled (Table 4).

\section{Logistic Regression Model}

A logistic regression model was constructed for the risk factor HAQ-DI and covariates on the outcome (WD). HAQDI, disease type and self-reported disease duration was acceptable to describe the outcome of WD in this cohort (Table 5a). In a subsequent analysis, the self-report variable of physically demanding work was included as an independent variable in the logistic regression model of HAQ-DI and covariates on WD (Table $\mathbf{5 b}$ ). Inclusion of the physically

Table 3. Demographics, Work Disability and HAQ-DI Scores in deSSc vs lcSSc

\begin{tabular}{|c|c|c|c|c|c|}
\hline & dcSSc & lcSSc & OR & $95 \%$ CI for OR & p-Value \\
\hline $\mathbf{N}$ & 26 & 35 & - & - & - \\
\hline Age & $51.65 \pm 1.97$ & $52.26 \pm 1.69$ & - & - & 0.82 \\
\hline Female sex & $73.1 \%$ & $94.3 \%$ & 0.16 & $0.03,0.87$ & 0.03 \\
\hline Less than high school education & $19.2 \%$ & $14.3 \%$ & 1.43 & $0.37,5.56$ & 0.73 \\
\hline HAQ-DI & $1.19 \pm 0.13$ & $0.88 \pm 0.11$ & - & - & 0.09 \\
\hline HAQ Pain ${ }^{a}$ & $1.31 \pm 0.16$ & $1.39 \pm 0.14$ & - & - & 0.72 \\
\hline Self-reported physically demanding work ${ }^{b}$ & $54.2 \%$ & $38.7 \%$ & 1.87 & $0.64,5.51$ & 0.29 \\
\hline
\end{tabular}

P-values provided for dichotomous data were obtained using Fisher's exact chi-squared tests. Odds ratio (OR) are provided for dcSSc relative to lcSSc. Continuous data were tested using independent t-tests assuming unequal variances.

${ }^{a}$ Data were missing for $2 \mathrm{dcSSc}$, and 2 lcSSc subjects; ${ }^{\mathbf{b}}$ Data were missing for $2 \mathrm{dcSSc}$, and 4 lcSSc subjects. 
Table 4. HAQ Scores and Demographic Factors for Those who Reported Work Disability (WD) vs No Work Disability (nWD) for Each Group of Subjects with Limited or Diffuse SSc

\begin{tabular}{|c|c|c|c|c|c|}
\hline & WD & nWD & OR & 95\% CI for OR & p-Value \\
\hline \multicolumn{6}{|l|}{ deSSc Only } \\
\hline Age & $53.2 \pm 2.88$ & $48.8 \pm 3.96$ & - & - & 0.45 \\
\hline Female sex & $82.4 \%$ & $55.6 \%$ & 3.73 & $0.61,22.86$ & 0.19 \\
\hline Less than high school education & $29.4 \%$ & $0 \%$ & & & 0.13 \\
\hline HAQ-DI & $1.34 \pm 0.15$ & $0.90 \pm 0.21$ & - & - & 0.073 \\
\hline HAQ Pain ${ }^{a}$ & $1.36 \pm 0.19$ & $1.21 \pm 0.26$ & - & - & 0.68 \\
\hline Self-reported physically demanding work ${ }^{b}$ & $62.5 \%$ & $37.5 \%$ & 2.78 & $0.48,16.03$ & 0.39 \\
\hline Female sex & $94.1 \%$ & $94.4 \%$ & 0.94 & $0.05,16.35$ & 1.00 \\
\hline Self-reported disease duration & $16.12 \pm 1.88$ & $10.50 \pm 1.83$ & - & - & 0.04 \\
\hline Less than high school education & $23.5 \%$ & $5.6 \%$ & 5.23 & $0.52,52.55$ & 0.18 \\
\hline HAQ-DI & $1.26 \pm 0.15$ & $0.53 \pm 0.14$ & - & - & 0.0016 \\
\hline HAQ Pain ${ }^{a}$ & $1.62 \pm 0.22$ & $1.19 \pm 0.20$ & - & - & 0.14 \\
\hline Self-reported physically demanding work ${ }^{b}$ & $47.1 \%$ & $28.6 \%$ & 2.22 & $0.50,9.96$ & 0.46 \\
\hline
\end{tabular}

P-values provided for dichotomous data were obtained using Fisher's exact chi-squared tests. Odds ratios (OR) have been provided for work disabled relative to employed. Continuous data were tested using independent t-tests assuming unequal variances.

${ }^{a}$ Data were missing for $2 \mathrm{dcSSc}$, and 2 lcSSc subjects; ${ }^{\mathrm{b}}$ Data were missing for $2 \mathrm{dcSSc}$, and $4 \mathrm{lcSSc}$ subjects.

demanding work factor altered the effect of the $1 \mathrm{cSSc}$ indicator variable in the model, but had little impact on other factors (Table 5). Thus, inclusion of this factor was useful in the model.

\section{Summary of Bivariate Analyses}

The proportion of subjects who reported being work disabled was significantly higher in the SSc group compared to the RA group $(55.7 \%$ vs $34.6 \%, \mathrm{p}=0.009)$. HAQ-DI was not significantly different between the SSc and RA groups. In both the SSc and RA groups, demographic variables were largely similar between those who were work disabled and those who were not. The exploratory variable, self-reported physically demanding work, was more common among work disabled cases (although not significantly) in all groups.

In the subset analysis of dcSSc $(n=26)$ compared to $1 c S S c$ $(n=35)$, those with lcSSc were significantly more likely to be female and have a longer disease duration. Exploratory analyses of work disabled vs not work disabled subjects within the dcSSc and $\mathrm{lcSSc}$ disease subsets revealed no differences between those with WD vs those without in the dcSSc group, suggesting that the analysis was underpowered. Disease duration was also significantly longer in work disabled lcSSc subjects $(\mathrm{p}=0.04)$.

In conclusion, it appears that higher HAQ-DI scores are strongly associated with WD in both SSc and RA. Although SSc patients have more WD compared to RA patients. The same trend of higher HAQ-DI in work disabled patients was apparent in the lcSSc subset, but no associations of any kind were observed in the dcSSc subset which may have been underpowered to detect any differences.

\section{DISCUSSION}

The proportion of self-reported WD in this southwestern Ontario cohort of SSc with mean disease duration of $11.02 \pm$ 1.22 years was $56 \%$. In the one other study by Moser et al. that reported the proportion of WD in an SSc study population, the estimate was lower than that observed here $(36 \%$; homemakers were included in the working group) [42]. Both estimates are high. Both studies had similar definitions of WD and similar study cohort characteristics, including mean age, proportion of females, and proportion which had completed at least high school; however, proportions of $\mathrm{dcSSc} / \mathrm{lcSSc}$ were not described in the Moser study [42]. Our study cohort had a longer mean disease duration (11 vs 8 years). This may partially account for the higher proportion with WD observed in the southwestern Ontario SSc cohort (as disease duration was independently associated with WD in a logistic regression model in this cohort, $\mathrm{p}=0.042$ ).

The estimate of WD in SSc we observed (as well as the Moser estimate) was relatively high and is comparable to (or higher than) WD in RA, where the large majority of RA studies have reported WD in at least $30 \%$ of subjects by 10 years disease duration (range 27-62\%). We compared WD in $\mathrm{SSc}$ with our internal RA reference group, and the propor- 
Table 5a. Reduced Logistic Regression Model of HAQ-DI, Disease Type, and Disease Duration on WD. Variables: Sex, Age, and 'Less than High School Education' were Removed from the Model

\begin{tabular}{|l|c|c|c|c|}
\hline \multicolumn{1}{|c|}{ Explanatory Variable (X) } & $\boldsymbol{\beta}_{\mathbf{X}}$ Estimate & Standard Error & Odds Ratio & Wald Test p-Value \\
\hline \hline $\mathbf{N}$ & 164 & - & - & - \\
\hline HAQ-DI score & 1.775 & 0.323 & 2.102 & $<0.001$ \\
\hline Disease type & & & 3.728 & 0.032 \\
Indicator IcSSc & 0.743 & 0.473 & 1.044 & 0.015 \\
Indicator dcSSc & 1.316 & 0.541 & 0.042 \\
\hline Self-reported disease duration & 0.043 & 0.021 & 0.053 & $<0.001$ \\
\hline Constant & -2.930 & 0.492 & \\
\hline
\end{tabular}

No statistically important collinearity between the HAQ-DI and any of the independent factors was observed.

Table 5b. Reduced Logistic Regression Model of HAQ-DI, Disease Type, Disease Duration and Self-Reported Physically Demanding Work on WD. Variables: Sex, Age, and 'Less than High School Education' were Removed from the Model

\begin{tabular}{|l|c|c|c|c|}
\hline \multicolumn{1}{|c|}{ Explanatory Variable $(\mathbf{X})$} & $\boldsymbol{\beta}_{\mathbf{X}}$ Estimate & Standard Error & Odds Ratio & Wald Test p-Value \\
\hline \hline N & 148 & - & - & - \\
\hline HAQ-DI score & 1.858 & 0.348 & 6.412 & $<0.001$ \\
\hline Disease type & & & 3.451 \\
Indicator lcSSc & 1.239 & 0.549 & 3.721 & 0.016 \\
Indicator dcSSc & 1.314 & 0.574 & 1.041 & 0.022 \\
\hline Self-reported disease duration & 0.041 & 0.022 & 3.062 & 0.069 \\
\hline Self-reported physically demanding work & 1.119 & 0.450 & 0.013 \\
\hline Constant & -3.565 & 0.654 & 0.028 & $<0.001$ \\
\hline
\end{tabular}

Equation: Logit $(\mathrm{p})=\ln [\mathrm{p} /(1-\mathrm{p})]=-2.930+1.775[\mathrm{HAQ}-\mathrm{DI}]+0.743[\mathrm{lcSSc}]+1.316[\mathrm{dcSSc}]+0.043$ [SR duration] where $\mathrm{p}=$ proportion with WD.

tions were significantly different $(56 \%$ vs $35 \%, \mathrm{p}=0.009)$ (Table 1). This difference in frequency may be partially due to the availability of more effective disease-modifying treatments in RA compared to SSc.

Despite the fact that this study used secondary data, the definition of WD was fairly consistent with those of several studies of WD in RA. This suggests that the measurement tool was accurate enough to adequately capture the relative impact of WD in SSc.

HAQ-DI scores were the most important independent factors associated with WD in both SSc and in RA. This is the first study to examine the relationship between HAQ-DI scores and WD in SSc; however, it is not surprising that this strong relationship exists, given that in the literature the HAQ-DI is strongly associated with WD in every RA study that assesses HAQ-DI [6, 9-13, 15-29].

Logistic regression analyses demonstrated that longer disease duration was marginally important in determining WD $\left(\beta_{\text {dur }}=0.043, \mathrm{OR}=1.044, \mathrm{p}=0.042\right)$; however, the effect of disease duration may be modified by disease extent.

Several studies have described more WD in women with RA than men [19, 20, 26], whereas others have not [13, 43]. Education has been associated with WD in RA. Highest level of education is often used as a proxy measure of SES [13, 17, 19, 20]. Disease duration of RA may affect WD but not in all literature [13].
High job demands, autonomy and control over workrelated factors, and support in the work place are recognized factors associated with WD. RA patients often need to decrease their hours and/or change jobs, although this may not prevent subsequent WD [44-46]. High physical requirements have been associated with WD in some studies $[4,6,7,9$, $13,15,19,22]$ but not in others [16, 17]. Anxiety and depression have been associated with current and subsequent WD in RA [10, 19, 20]. Danieli et al. reported that people with SSc in their cohort had more depressive symptoms compared to those with RA [37]. Thus, it is feasible that the psychological burden of SSc is substantial and may be associated with WD, although these factors were not addressed in this study.

One study of psychological adjustment in SSc reported proportions of work disabled study participants [42]. Of 94 respondents to a mailed questionnaire (64\% response rate), $23(24.5 \%)$ were work disabled, $24(25.5 \%)$ were working part-time or full-time outside the home, $17(18.1 \%)$ were homemakers, and 27 (28.7\%) were retired [42].

The population of southwestern Ontario has unique characteristics compared to other regions in Ontario. London is an urban center in which households may tend to have moderate-high SES (a common proxy measure for SES, having at least a high school education, was common in both SSc [83.6\%] and RA [90.3\%] respondents). Rural areas in the region may have less high SES households and different practices with respect to occupations, and some patients with SSc travel from remote geographic locations to receive care 
at the London clinic. Due to the unique catchment area serviced by the London rheumatology clinic, the results of this study may not be generalizable beyond southwestern Ontario, or even the study rheumatologist's cohort. However, it is likely that results were representative of SSc and RA patients referred to rheumatology services in southwestern Ontario. Additional research would improve the certainty of this statement.

A limitation to this study is that it is cross-sectional (involving the analysis of data collected for another study with linkage to HAQs completed around the same time). Thus, there is a lack of information about the temporal association of potential risk factors (including disease/symptom onset and time of earliest high HAQ-DI scores) and the outcome. The distinction between the proportion with WD and the risk of developing WD is important. An additional limitation to this research was that subset analyses conducted between $\mathrm{dcSSc}$ and $\mathrm{lcSSc}$ subsets were underpowered, and thus no conclusions can be drawn based on these data. Strengths of this study include that the participants were well characterized (who met criteria for dcSSc, lcSSc, or RA), comparison groups were used appropriately, and the HAQDI was used as the outcome measure, which is standardized and validated in SSc.

The prevention of WD may be more effective than correction following job loss [15] and vocational programs often intervene too late [27]. Assessment tools to determine the need for job accommodation that may identify the problems, and thus allow postponement or prevention of work loss with timely intervention (or that have been designed to develop a rehabilitation plan) have been developed for other MSK conditions $[44,47]$. It is feasible that a similar tool modified to specifically address future development of WD in SSc could perhaps be useful in the future.

\section{CONCLUSIONS}

The frequency of WD in SSc is high (56\%) and is greater than in RA. SSc, and in particular, dcSSc have significantly more WD than RA. The HAQ-DI is strongly associated with WD in SSc, OR = 5.902 (as well as in RA). Implications of this study include a strong case for continued research in WD in SSc (including prediction of subsequent WD by HAQ-DI scores), and perhaps the eventual development of timely and important work-based interventions specific to SSc.

\section{REFERENCES}

[1] Seibold J. Connective tissue diseases characterized by fibrosis. In: Ruddy S, Harris ED, Jr., Sledge CB, Eds., Textbook of Rheumatology Vol. 2. Philadelphia: WB Saunders Co., 2001: 1133-59.

[2] Wigley F. Systemic Sclerosis. B. Clinical Features. In: Klippel JH, Ed., Primer on the Rheumatic Diseases. Atlanta: The Arthritis Foundation 2001: 357-64.

[3] Smyth AE, MacGregor AJ, Mukerjee D, Brough GM, Black CM, Denton CP. A cross-sectional comparison of three self-reported functional indices in scleroderma. Rheumatology (Oxford) 2003; 42: 732-8.

[4] Yelin E, Meenan R, Nevitt M, Epstein W. Work disability in rheumatoid arthritis: effects of disease, social, and work factors. Ann Intern Med 1980; 93: 551-6.

[5] Meenan RF, Yelin EH, Nevitt M, Epstein WV. The impact of chronic disease: a sociomedical profile of rheumatoid arthritis. Arthritis Rheum 1981; 24:544-9.
Makisara GL, Makisara P. Prognosis of functional capacity and work capacity in rheumatoid arthritis. Clin Rheumatol 1982; 1: 117-25.

[7] Kaarela K, Lehtinen K, Luukkainen R. Work capacity of patients with inflammatory joint diseases. An eight-year follow-up study. Scand J Rheumatol 1987; 16: 403-6.

[8] Yelin E, Henke C, Epstein W. The work dynamics of the person with rheumatoid arthritis. Arthritis Rheum 1987; 30: 507-12.

[9] Reisine ST, Grady KE, Goodenow C, Fifield J. Work disability among women with rheumatoid arthritis. The relative importance of disease, social, work, and family factors. Arthritis Rheum 1989; 32:538-43.

[10] Borg G, Allander E, Berg E, Brodin U, From A, Trang L. Auranofin treatment in early rheumatoid arthritis may postpone early retirement. Results from a 2-year double blind trial. J Rheumatol 1991; 18: 1015-20.

[11] Callahan LF, Bloch DA, Pincus T. Identification of work disability in rheumatoid arthritis: physical, radiographic and laboratory variables do not add explanatory power to demographic and functional variables. J Clin Epidemiol 1992; 45: 127-38.

[12] Eberhardt K, Larsson BM, Nived K. Early rheumatoid arthritis-some social, economical, and psychological aspects. Scand J Rheumatol 1993; 22: 119-23.

[13] Doeglas D, Suurmeijer T, Krol B, Sanderman R, van Leeuwen M, van Rijswijk M. Work disability in early rheumatoid arthritis. Ann Rheum Dis 1995; 54: 455-60.

[14] Reisine S, McQuillan J, Fifield J. Predictors of work disability in rheumatoid arthritis patients. A five-year followup. Arthritis Rheum 1995; 38: 1630-7.

[15] Allaire SH, Anderson JJ, Meenan RF. Reducing work disability associated with rheumatoid arthritis: identification of additional risk factors and persons likely to benefit from intervention. Arthritis Care Res 1996; 9: 349-57.

[16] Mau W, Bornmann M, Weber H, Weidemann HF, Hecker H, Raspe HH. Prediction of permanent work disability in a follow-up study of early rheumatoid arthritis: results of a tree structured analysis using RECPAM. Br J Rheumatol 1996; 35: 652-9.

[17] Fex E, Larsson BM, Nived K, Eberhardt K. Effect of rheumatoid arthritis on work status and social and leisure time activities in patients followed 8 years from onset. J Rheumatol 1998; 25: 44-50.

[18] Van Jaarsveld CH, Jacobs JW, Schrijvers AJ, van Albada-Kuipers GA, Hofman DM, Bijlsma JW. Effects of rheumatoid arthritis on employment and social participation during the first years of disease in The Netherlands. Br J Rheumatol 1998; 37: 848-53.

[19] Wolfe F, Hawley DJ. The longterm outcomes of rheumatoid arthritis: Work disability: a prospective 18 year study of 823 patients. J Rheumatol 1998; 25: 2108-17.

[20] De Roos AJ, Callahan LF. Differences by sex in correlates of work status in rheumatoid arthritis patients. Arthritis Care Res 1999; 12: 381-91.

[21] Jantti J, Aho K, Kaarela K, Kautiainen H. Work disability in an inception cohort of patients with seropositive rheumatoid arthritis: a 20 year study. Rheumatology (Oxford) 1999; 38: 1138-41.

[22] Sokka T, Kautiainen H, Mottonen T, Hannonen P. Work disability in rheumatoid arthritis 10 years after the diagnosis. J Rheumatol 1999; 26: 1681-5.

[23] Barrett EM, Scott DG, Wiles NJ, Symmons DP. The impact of rheumatoid arthritis on employment status in the early years of disease: a UK community-based study. Rheumatology (Oxford) 2000; 39: 1403-9.

[24] Newhall-Perry K, Law NJ, Ramos B, et al. Direct and indirect costs associated with the onset of seropositive rheumatoid arthritis. Western Consortium of Practicing Rheumatologists. J Rheumatol 2000; 27: 1156-63.

125] Chorus AM, Miedema HS, Wevers CW, van der Linden S. Work factors and behavioural coping in relation to withdrawal from the labour force in patients with rheumatoid arthritis. Ann Rheum Dis 2001; 60: 1025-32.

[26] Young A, Dixey J, Kulinskaya E, et al. Which patients stop working because of rheumatoid arthritis? Results of five years' follow up in 732 patients from the Early RA Study (ERAS). Ann Rheum Dis 2002; 61: 335-40.

[27] Lacaille D, Sheps S, Spinelli JJ, Chalmers A, Esdaile JM. Identification of modifiable work-related factors that influence the risk of work disability in rheumatoid arthritis. Arthritis Rheum 2004; 51: 843-52. 
[28] Puolakka K, Kautiainen H, Mottonen T, et al. Early suppression of disease activity is essential for maintenance of work capacity in patients with recent-onset rheumatoid arthritis: five-year experience from the FIN-RACo trial. Arthritis Rheum 2005; 52:36-41.

[29] Verstappen SM, Boonen A, Bijlsma JW, et al. Working status among Dutch patients with rheumatoid arthritis: work disability and working conditions. Rheumatology (Oxford) 2005; 44: 202-6.

[30] Gordon DA, Hastings DE. Clinical features of rheumatoid arthritis. In: Hochberg MC, Silman AJ, Smolen JS, Weinblatt ME, Weisman MH, Eds., Rheumatology Vol. 1. Toronto: Elsevier Limited (Mosby), 2003: 765-70.

[31] Fries JF, Spitz P, Kraines RG, Holman HR. Measurement of patient outcome in arthritis. Arthritis Rheum 1980; 23: 137-45.

[32] Bruce B, Fries JF. The Stanford Health Assessment Questionnaire: a review of its history, issues, progress, and documentation. J Rheumatol 2003; 30: 167-78.

[33] van Riel PLCM, van Gestel AM, Welsing PMJ. In: Hochberg MC, Silman AJ, Smolen JS, Weinblatt ME, Weisman MH, Eds., Rheumatology Vol. 1. Toronto: Elsevier Limited (Mosby), 2003: 900-1.

[34] Ollier WE, Harrison B, Symmons D. What is the natural history of rheumatoid arthritis? Best Pract Res Clin Rheumatol 2001; 15: 2748.

[35] Steen VD, Medsger TA, Jr. The value of the Health Assessment Questionnaire and special patient-generated scales to demonstrate change in systemic sclerosis patients over time. Arthritis Rheum 1997; 40: 1984-91.

[36] Clements PJ, Wong WK, Hurwitz EL, et al. Correlates of the disability index of the health assessment questionnaire: a measure of functional impairment in systemic sclerosis. Arthritis Rheum 1999;42(11): 2372-80.

[37] Danieli E, Airo P, Bettoni L, et al. Health-related quality of life measured by the Short Form 36 (SF-36) in systemic sclerosis: correlations with indexes of disease activity and severity, disability, and depressive symptoms. Clin Rheumatol 2005; 24: 48-54.
[38] Hans GK, Pope J. Validity of a self-report questionnaire about osteoporosis used in a case control study of subjects with scleroderma, rheumatoid arthritis, and non-inflammatory musculoskeletal disease. [Abstract]. J Rheumatol 2004; 31: 1446.

[39] Subcommittee for scleroderma Criteria of the American Rheumatism Association Diagnostic and Therapeutic Criteria Committee. Preliminary criteria for the classification of systemic sclerosis (scleroderma). Arthritis Rheum 1980; 23: 581-91.

[40] LeRoy EC, Black C, Fleischmajer R, et al. Scleroderma (systemic sclerosis): classification, subsets and pathogenesis. J Rheumatol $1988 ; 15: 202-5$.

[41] Arnett FC, Edworthy SM, Bloch DA, et al. The American Rheumatism Association 1987 revised criteria for the classification of rheumatoid arthritis. Arthritis Rheum 1988; 31: 315-24.

[42] Moser DK, Clements PJ, Brecht ML, Weiner SR. Predictors of psychosocial adjustment in systemic sclerosis. The influence of formal education level, functional ability, hardiness, uncertainty, and social support. Arthritis Rheum 1993; 36: 1398-405.

[43] Albers JM, Kuper HH, van Riel PL, et al. Socio-economic consequences of rheumatoid arthritis in the first years of the disease. Rheumatology (Oxford) 1999; 38: 423-30.

[44] Allaire SH. Update on work disability in rheumatic diseases. Curr Opin Rheumatol 2001; 13: 93-8.

[45] Lacaille D. Arthritis and employment research: where are we? Where do we need to go? J Rheumatol Suppl 2005; 72: 42-5.

[46] Verstappen SM, Bijlsma JW, Verkleij H, et al. Overview of work disability in rheumatoid arthritis patients as observed in crosssectional and longitudinal surveys. Arthritis Rheum 2004; 51: 48897.

[47] Durand MJ, Loisel P, Hong QN, Charpentier N. Helping clinicians in work disability prevention: the work disability diagnosis interview. J Occup Rehabil 2002; 12: 191-204. 